Arq. Bras. Med. Vet. Zootec., v.70, n.2, p.486-490, 2018

\title{
Gota úrica visceral em bobo-pequeno (Puffinus puffinus) no sul do Brasil
}

\author{
[Visceral gout uric in manx shearwater (Puffinus puffinus) in Southern of Brazil] \\ D. Fink ${ }^{1,2}$, L. Drumond ${ }^{1}$, M.I. Basílio ${ }^{1}$, C.M. Sartori ${ }^{1}$, T.R. Andrade ${ }^{1,2}$, N.Z. Santos ${ }^{1}$, M.J. Cremer ${ }^{1,2}$ \\ ${ }^{1}$ Universidade da Região de Joinville - Unidade de Estabilização de Animais Marinhos - Laboratório de Ecologia e \\ Conservação de Tetrápodes Marinhos e Costeiros - São Francisco do Sul, SC \\ ${ }^{2}$ Programa de pós-graduação em Saúde e Meio Ambiente - Joinville, SC
}

\section{RESUMO}

A gota úrica visceral é uma doença que acomete répteis, aves e mamíferos. Caracteriza-se por depósitos de cristais de urato e ácido úrico em diferentes órgãos da região visceral. O objetivo deste trabalho foi relatar um caso de gota úrica visceral em um indivíduo de bobo-pequeno (Puffinus puffinus) encontrado morto no litoral norte de Santa Catarina, sul do Brasil. No período de 20 de agosto de 2015 a 20 de abril de 2016, as praias dos municípios de Araquari, Barra do Sul, São Francisco do Sul e Itapoá foram monitoradas diariamente para o registro e a recuperação de tetrápodes marinhos mortos, incluindo aves marinhas. Foram encontrados e necropsiados 84 indivíduos. Um deles apresentou o pericárdio aderido ao miocárdio e com a coloração esbranquiçada. Os rins, o fígado e os pulmões continham inúmeros pontos esbranquiçados. A ocorrência dessa patologia na espécie foi de 1,19\%. Trata-se do primeiro relato de bobo-pequeno com gota úrica visceral encontrado no Brasil.

Palavras-chave: gota úrica visceral, Brasil, Puffinus puffinus

\begin{abstract}
Visceral gout uric is a disease that affects reptiles, birds and mammals. It is characterized by urate crystal deposits and uric acid in different organs of visceral region. The objective of this study was to report a case of visceral urica drop in a manx shearwater individual (Puffinus puffinus) found dead on the north coast of Santa Catarina, Southern Brazil. In the period from 20 August 2015 and 20 April 2016, beaches in the municipalities of Araquari, Barra do Sul, São Francisco do Sul and Itapoá, were monitored daily for the registration and recovery of dead marine tetrapods, including seabirds. Eightyfour were found and necropsied. One of them was whitish in color and had the pericardium adhered to the myocardium. The kidneys, liver and lungs contained numerous whitish dots. The occurrence of this disease in the species was $1.19 \%$. This is the first manx shearwater report with visceral urica drop found in Brazil.
\end{abstract}

Keywords: visceral gout uric, Brazil, Puffinus puffinus

\section{INTRODUÇÃO}

Nas aves, os resíduos nitrogenados são excretados na forma de ácido úrico (Del Hoyo et al., 1992; Carciofi e Oliveira, 2006). Quando há grande quantidade de nitrogênio no organismo, falhas renais podem ser geradas pelo acúmulo de acido úrico, causando hiperuricemia ou gota úrica (Lumeij et al., 1998; Coppola et al., 2013).

A gota úrica é uma patologia metabólica, caracterizada pela deposição de cristais de urato e ácido úrico, que não são excretados adequadamente pelos rins, sendo conhecida nas formas visceral e articular (Lierz, 2003; Doneley, 2010). Na forma articular, verifica-se um aumento do volume e sensibilidade nas articulações devido à deposição de cristais dentro das cápsulas sinoviais e nas bainhas dos tendões (Burgos-Rordriguez, 2010). Essa forma é comumente encontrada em mamíferos, principalmente humanos (Coelho, 2002). Na forma visceral, dificilmente se observam sinais clínicos e o diagnóstico geralmente é realizado

Recebido em 24 de março de 2017

Aceito 24 de maio de 2017

E-mail:dani.fink@gmail.com 
na necropsia (Pollock, 2006). Os cristais de urato são depositados nas superfícies serosas do pericárdio, do fígado, do baço e dentro do rim, mas podem estar presentes em qualquer tecido (Lierz, 2003; Pollock, 2006).

Inúmeros fatores predisponentes estão associados à doença: insuficiência renal, escassez hídrica, predisposição genética, deficiência de vitamina A, excesso de proteína e cálcio na dieta (Pollock, 2006; Doneley, 2010; Costa et al., 2015) e exposição a diclofenaco (Shultz et al., 2004). A forma visceral geralmente é aguda, e a articular crônica, e raramente as duas formas ocorrem simultaneamente (Godoy, 2006).

As aves marinhas compreendem um grupo diversificado de espécies que vivem associadas aos ecossistemas marinhos, alimentando-se de organismos de água salgada (Schreiber e Burger, 2002). A ordem Procellariiformes abrange aves marinhas oceânicas ou pelágicas das famílias Diomedeidae (albatrozes) e Procellariidae (petréis, pardelas e bobos). $\mathrm{O}$ bobo-pequeno (Puffinus puffinus) é uma espécie gregária e migratória transequatorial, ou seja, migra anualmente entre a Europa e a América do Sul (Del Hoyo et al., 1992; Sick, 1997). As colônias encontram-se no Atlântico Norte (acima de $30^{\circ} \mathrm{N}$ ), onde ele se reproduz de maio a setembro. Nos meses de setembro a fevereiro, alcança o litoral brasileiro (Sick, 1997). Sua dieta consiste de pequenos peixes e cefalópodes (Del Hoyo et al., 1992).

Devido a sua posição de predadores na cadeia trófica, essas aves tornam-se suscetíveis à bioacumulação de poluentes e, por responderem a alterações na oferta de alimentos, são consideradas biondicadores de poluição no ambiente marinho e estuarino (Furness e Camphuysen, 1997; Burger e Gochfield, 2004). Consequentemente, em razão das longas rotas migratórias, contribuem para a compreensão dos impactos no ecossistema marinho em escala global (Shaeffer et al., 2006; Guilford et al., 2009).

O objetivo deste trabalho foi relatar um caso de gota úrica visceral em um indivíduo de bobopequeno ( $P$. puffinus) encontrado morto no litoral de Santa Catarina, sul do Brasil, assim como apresentar a ocorrência dessa patologia entre os indivíduos que chegam a essa faixa do litoral.

\section{MATERIAL E MÉTODOS}

No período de 25 de agosto de 2015 a 20 de abril de 2016, as praias dos municípios de Araquari, Barra do Sul, São Francisco do Sul e Itapoá, no litoral norte de Santa Catarina (26³4'94''S $48^{\circ} 39^{\prime} 50^{\prime \prime} \mathrm{O}$ até $25^{\circ} 58^{\prime} 36^{\prime \prime} \mathrm{S} \quad 48^{\circ} 35^{\prime} 45^{\prime \prime} \mathrm{O}$ ), foram monitoradas diariamente para o registro e a recuperação de tetrápodes marinhos mortos, incluindo aves marinhas. $\mathrm{O}$ monitoramento foi realizado a pé, de bicicleta ou com veículos, percorrendo toda a linha de praia, numa extensão de $81 \mathrm{~km}$. Para todos os indivíduos encontrados, foi registrada a coordenada, a data, a hora e a espécie. Todos os exemplares encontrados foram transportados para laboratório, onde passaram por um procedimento padrão de necropsia previamente estabelecido, realizado por veterinários. O registro da biometria básica (comprimento do membro posterior, asa cotovelo, total asa, tarso, comprimento corporal total, bico, altura e largura do bico e peso total) (adaptado de Sick, 1997) e a necropsia foram realizados, sempre que possível, na mesma data de coleta do indivíduo. As medidas foram feitas com paquímetro e régua. Para animais em bom estado de conservação, foram coletados fragmentos de órgãos macroscopicamente alterados. Estes foram acondicionados em formol $10 \%$ e enviados para análise ao LAPCOM Laboratório de Patologia Comparada de Animais Selvagens da Faculdade de Medicina Veterinária e Zootecnia da Universidade de São Paulo, onde foram processados seguindo protocolo de rotina para histologia e corados pela hematoxilina e eosina (HE).

A ocorrência dessa patologia foi calculada para a região como sendo a proporção entre o número de indivíduos acometidos pela doença em relação ao número de indivíduos da espécie encontrados mortos na faixa de litoral monitorada em um determinado intervalo de tempo, multiplicado por 100 .

\section{RESULTADOS}

Foram registrados e necropsiados 84 bobos-pequenos (Puffinus puffinus) ao longo de oito meses de monitoramento. Dentre estes, apenas um indivíduo apresentou alterações 
macroscópicas compatíveis com gota úrica visceral. O exemplar foi encontrado na praia do Pontal, município de Itapoá $\left(26^{\circ} 177^{\prime} 02 ”\right.$ S 48 589'80” O), no dia 08 de outubro de 2015. Era um macho adulto, com a carcaça em bom estado de conservação, que apresentou as seguintes medidas: comprimento do membro posterior $=7,21 \mathrm{~cm}$; comprimento total da asa $=$ $29 \mathrm{~cm}$; comprimento do $\operatorname{tarso}=4,2 \mathrm{~cm}$; comprimento corporal total $=36 \mathrm{~cm}$; comprimento do bico $=2,8 \mathrm{~cm}$; altura do bico $=0,4 \mathrm{~cm}$; largura do bico $=0,5 \mathrm{~cm}$. O peso do animal era de $385 \mathrm{~g}$. Considerando o número de indivíduos da espécie registrados no período, a ocorrência da gota úrica visceral em indivíduos de bobo-pequeno que encalharam mortos no litoral norte de Santa Catarina foi estimada em $1,19 \%$.

As alterações foram visualizadas no coração, nos rins, nos pulmões e no fígado. O pericárdio estava esbranquiçado (depósito de urato), hipertrofiado e aderido ao miocárdio (Fig. 1A). Pequenos sinais de depósito de urato também foram vistos no fígado e nos pulmões (Fig. 1B). Os rins apresentavam-se hipertrofiados, com aspecto granular branco-acinzentado (Fig. 1C).
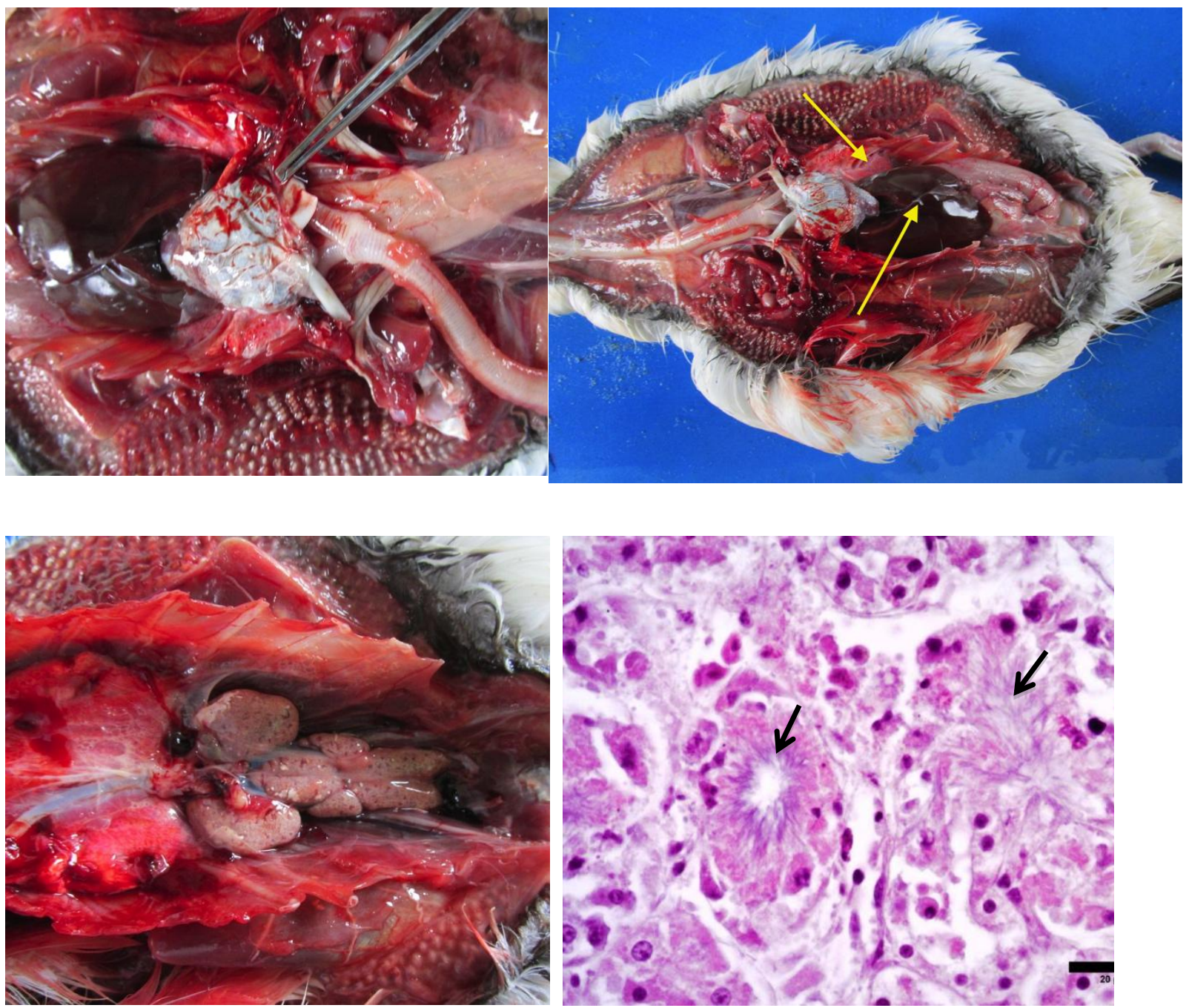

Figura 1. Cavidade celomática do exemplar de Puffinus puffinus diagnosticado com gota úrica visceral no litoral norte de Santa Catarina. A) pericárdio aderido ao miocárdio; B) depósito de urato no fígado e no pulmão; C) rins com aspecto granular; D) rim, com discreta dilatação do túbulo com presença de material amorfo no lúmen (setas), HE. 
$\mathrm{Na}$ análise microscópica, o fígado apresentou congestão. Os rins apresentaram degeneração multifocal discreta, com a presença de múltiplos agregados nodulares corticais, pouco delimitados, não encapsulados, compostos por deposição de material amorfo eosinofílico circundado por um discreto infiltrado inflamatório heterofílico e histiocístico (Fig. 1D).

\section{DISCUSSÃO}

As lesões encontradas no bobo-pequeno foram a provável causa do óbito do animal, pois estas podem ter provocado o colapso dos principais órgãos. Todas as características macro e microscópicas observadas no bobo-pequeno corroboram as alterações relatadas na literatura para gota úrica visceral em aves (Coppola et al., 2013; Sales et al., 2015). Contudo, não foi possível identificar a origem da doença.

Apesar de $P$. puffinus ser encontrado pontualmente no nordeste do Brasil (Ceará, Rio Grande do Norte e Bahia) e continuamente do sudeste ao sul do litoral brasileiro (Sick, 1997), não há registros na literatura de gota úrica visceral na espécie, assim como para a ordem Procellariiformes. A ausência de registros dessa doença em aves marinhas contribui para a dificuldade em se encontrar um fator predisponente.

Para aves de produção, o excesso de cálcio na alimentação é considerado a principal causa de ocorrência dessa doença (Coelho, 2002). Porém, a patogênese e a etiologia da gota úrica não são totalmente compreendidas e podem estar associadas à desidratação, à obesidade, ao estresse, ao excesso de cálcio e proteína na dieta, à deficiência de vitamina $\mathrm{A}$ ou ao desbalanço de aminoácidos (Pollock, 2006; Carciofi e Oliveira, 2006).

A doença já foi descrita para algumas espécies de aves brasileiras. Sales et al. (2015) fizeram o primeiro relato de gota úrica visceral em uma espécie de rapinante de vida livre, a coruja suindara (Tyto alba). Os autores indicaram a patologia como responsável pela morte do indivíduo, devido à pericardite, à peri-hepatite e à nefrite diagnosticadas na microscopia. Da mesma forma, a doença foi encontrada em um tucano-toco (Ramphastos toco) de vida livre (Coppola et al., 2013) e em um gavião-real
(Harpia harpyja) de cativeiro (Costa et al., 2012). Entretanto, em ambos os casos, a causa da gota úrica visceral não foi elucidada. A gota úrica visceral costuma ser diagnosticada durante os procedimentos de necrópsia e confirmada com exames histológicos (Lierz, 2003; Pollock, 2006; Costa et al., 2012).

A análise de carcaças de animais mortos encontrados nas praias constitui uma importante estratégia para avaliar o estado de saúde dos oceanos, requerendo, no entanto, uma base de dados de longo prazo que possibilite avaliar variações na prevalência das patologias registradas.

\section{CONCLUSÃO}

Os resultados macroscópicos encontrados no bobo-pequeno, juntamente com as análises microscópicas, são compatíveis com as características encontradas no quadro de gota úrica visceral em aves. Este é o primeiro relato de gota úrica visceral em bobo-pequeno no sul do Brasil. Estudos mais aprofundados em aves marinhas são necessários para uma melhor avaliação da susceptibilidade, bem como a ocorrência da patologia em diferentes espécies.

\section{AGRADECIMENTOS}

Á equipe do LAPCOM-FMVZ-USP, em especial Pedro Navas-Suárez pela fotomicrografia.

\section{REFERÊNCIAS}

BURGOS-RODRÍGUEZ, A.G. Avian renal system: clinical implication. Vet. Clin. Exot. Anim., v.13, p.393-411, 2010.

BURGER, J.; GOCHFELD, M. Marine birds as sentinels of environmental pollution. EcoHealth., n. 1, p. 263-274, 2004.

CARCIOFI, A.C.; OLIVEIRA, L.D. Doenças nutricionais. In: CUBAS, Z.S.; SILVA, J.C.R.; CATÃO-DIAS, J.L. (Eds.). Tratado de animais selvagens: medicina veterinária. São Paulo: Roca, 2006. p.838-864.

COELHO, H.E. Patologia veterinária. Barueri: Manole, 2002. 234p. 
COPPOLA, M.P.; RODRIGUES, J.C.Z.; BAPTISTA, A.A.; SEQUEIRA, J.L. et al. Gota úrica visceral em tucano toco (Ramphastos toco). Vet. Zootec., v.20, p.260-263, 2013.

COSTA, A.M.; IMBELONI, A.A.; FERREIRA, V.L.; RASO, T.F. Gota úrica visceral em Harpia (Harpia harpyja). Nosso Clin., n.86, p.60-62, 2012.

DEL HOYO, J.; ELLIOT, A.; SARGATAL, J. (Eds.). Handbook of the birds of the world. Barcelona: Lynx Edicions, 1992. v.1, 696p.

DONELEY, B. Avian medicine and surgery in practice. London: Manson Publishing, 2010. 336p.

FURNESS, R.W.; CAMPHUYSEN, C.J. Seabirds as monitors of the marine environment. J. Mar. Sci., v.54, p.726-737, 1997.

GODOY, S.N. Psittaciformes (arara, papagaio, periquito). In: CUBAS, Z.S.; SILVA, J.C.R.; CATÃO-DIAS, J.L. (Eds.). Tratado de animais selvagens: medicina veterinária. São Paulo: Roca, 2006. p.222-251.

GUILFORD, T.; MEADE, J.; WILLIS, J.; PHILLIPS, R.A. et al. Migration and stopover in a small pelagic seabird, the Manx shearwater Puffinus puffinus: insights from machine learning. Proc. R. Soc., v.276. p.1215-1223, 2009.

LIERZ, M. Avian renal disease: pathogenesis, diagnosis, and therapy. Vet. Clin. Exot. Anim., v.6, p.29-55, 2003.
LUMEIJ, J.T.; SPRANG, E.P.M.; REDIG, P.T. Further studies on allopurinol-induced hyperuricaemia and visceral gout in red-tailed hawks (Buteo jamaicensis). Avian Pathol., v.27, p.390-393, 1998.

POLLOCK, C. Diagnosis and treatment of avian renal disease. Vet. Clin. Exot. Anim., v.9, p.107128, 2006.

SALES, I.S.; NOGUEIRA, C.H.O.; SILVEIRA, L.S. Gota úrica visceral em coruja suindara (Tyto alba) de vida livre. Pesqui. Vet. Bras., v.35, p.169-172, 2015.

SCHREIBER, E.A.; BURGER, J. (Eds.). Biology of marine birds. Boca Raton: CRC PRESS, 2002. $722 \mathrm{p}$.

SHAFFER, S.A.; TREMBLAY, Y.; WEIMERSKIRCH, H.; SCOTT, D. et al. Migratory shearwaters integrate oceanic resources across the Pacific Ocean in an endless summer. Proc. Nat. Acad. Sci. EUA, v.103, p.12799-12802, 2006.

SHULTZ, S.; BARAL, H.S.; CHARMAN, S.; CUNINNGHAM, A. et al. Diclofenac poisoning is widespread in declining vulture populations across the Indian subcontinent. Proc. R. Soc. B, v 271, Suppl.6, p.S458-S460, 2004.

SICK, H. Ornitologia brasileira. Rio de Janeiro: Nova Fronteira, 1997. 912p. 